This material is posted here with permission of the IEEE. Such permission of the IEEE does not in any way imply IEEE endorsement of any of BTH's products or services Internal or personal use of this material is permitted. However, permission to reprint/republish this material for advertising or promotional purposes or for creating new collective works for resale or redistribution must be obtained from the IEEE by sending a blank email message to pubs-permissions@ieee.org. By choosing to view this document, you agree to all provisions of the copyright laws protecting it.

\title{
AN IMPROVED ARRAY INTERPOLATION APPROACH TO DOA ESTIMATION IN CORRELATED SIGNAL ENVIRONMENTS
}

\author{
B. K. Lau', G. J. Cook ${ }^{2}$ and Y. H. Leung ${ }^{2}$ \\ ${ }^{1}$ ITS, Blekinge Institute of Technology, Sweden \\ ${ }^{2}$ WATRI, Curtin University of Technology, Australia
}

\begin{abstract}
Many popular direction-of-arrival (DOA) estimators rely on the fact that the array response vector of the array is Vandermonde, for example, that of a uniform linear array (ULA). Array interpolation is a preprocessing technique to transform the array response vector of a planar array of arbitrary geometry to that of a ULA over an angular sector. While good approximation within the target sector is attained in the existing array interpolation approaches, the response of the interpolated array in the out-ofsector region is at best partially controlled. Accordingly, out-ofsector signals, especially those highly correlated with the insector signals, can degrade significantly the performance of DOA estimators (e.g., MUSIC with spatial smoothing) that rely on the Vandermonde form to work correctly. In this paper, we propose an improved array interpolation approach that takes into account the array response over the full azimuth. We present also numerical examples to demonstrate the shortcomings of the existing approaches and the effectiveness of our proposal.
\end{abstract}

\section{INTRODUCTION}

Since the inception of array signal processing, uniform linear arrays (ULAs) have received by far the most attention. This is due in large part to their uniform spatial sampling which results in their array response vectors having a Vandermonde form. This form is central to the derivation of many important DOA estimators such as root-MUSIC [1], MUSIC with spatial smoothing [2], and root-WSF [3].

ULAs are, however, not always used in practice. Practical considerations such as hardware cost and the size and shape of the mounting platform [4],[5] can restrict the choice of array geometry. Moreover, ULAs cannot provide $360^{\circ}$ of coverage in the azimuthal plane which is necessary in many applications such as radar, sonar and wireless communications.

To provide $360^{\circ}$ of coverage, one has to deploy planar arrays such as uniform circular arrays (UCAs). However, the array response vector of a planar array is, in general, not Vandermonde. A number of techniques have been proposed to transform this vector to Vandermonde form. One such class of techniques is array interpolation, first proposed by Bronez [6], and later under different formulations by Friedlander [4] and Pesavento et al. [7]. In array interpolation, the array response vector of the planar array is mapped using a transformation matrix to that of a ULA over an angular sector in the azimuth, called the in-sector. Thus the approach involves sector-by-sector processing to cover the full azimuth [4]. Array interpolation has been combined with different DOA estimators, for example, root-MUSIC [4] with spatial smoothing in [8], ESPRIT in [9], MODE (or root-WSF) in [10], and PR-JES in [11]. Recently, the successful application of array interpolation to seismic detection [12] also helped establish its practicality.

In the work of Bronez [6], the transformation matrix is derived by minimizing the total response of the interpolated array under the constraint that the transformed array response vector matches the ULA array response vector over a grid of angles within the in-sector. Thus the out-of-sector response is reduced but not totally suppressed. In contrast, in the works of Friedlander et al., see for example [4], [5], and [7]-[10], the transformation matrix is found as the LS solution that best maps the array response vector of the planar array to that of a ULA for a finite set of angles within the in-sector. Although this method is simple, it is intuitively incomplete as it neglects the out-ofsector response. More recently, Pesavento et al. [7] pointed out that the out-of-sector response should not be neglected. They proposed a different formulation to attenuate the out-of-sector response to a preset level while minimizing the error in the insector. Their formulation can thus be regarded as a more refined version of the Bronez formulation. Pesavento et al. showed that their formulation can be written as a second order cone (SoC) problem which allows it to be solved efficiently using the SeDuMi MATLAB toolbox [13]. While this formulation can produce better results than that of Friedlander [4], it must allow for a roll-off region where the response is unaccounted for.

Other related developments include the recent works of Hyberg et al. [14] and Bühren et al. [15]. The aim of both methods is to reduce the bias in the DOA estimates which is an artifact of the Friedlander formulation. In [14], the authors cleverly incorporate the performance of the DOA estimator into the design of the transformation matrix, while in [15], the authors proposed the use of shift-invariance transformations to take advantage of the search-free ESPRIT algorithm and to circumvent the need for the interpolated array to be a physical array. Whilst both methods will reduce bias, like Friedlander, they also ignore the out-of-sector response.

In this paper, we show that DOA estimation with the Friedlander and Pesavento formulations can yield poor results when the received signals are highly correlated and are not all confined to the in-sector. This is due to the fact that, just outside the in-sector, the gain of the interpolated array is still significant while the phase response has deviated significantly from being Vandermonde. DOA estimators that rely on the Vandermonde 
form may thus not be able to deal with received signals in this region properly. We propose in this paper a new formulation to address this limitation. In particular, the new formulation deals explicitly with the entire out-of-sector region by setting a target response for this region in addition to the target response for the in-sector region. Here, we use MUSIC with spatial smoothing to visualize the impact of out-of-sector response on DOA estimation of in-sector signals, though in practice root-MUSIC [4] is more suited because it gives better performance.

\section{SIGNAL AND ARRAY MODELS}

Consider a planar array with $N$ elements. The $n$th component of the array response vector $\mathbf{a}(\theta), n=1, \ldots, N$, to a narrowband signal of wavelength $\lambda$ arriving from azimuth angle ${ }^{1}$ $\theta \in[-\pi, \pi]$ is given by

$$
a_{n}(\theta)=G_{n}(\theta) \exp \left[j k\left(x_{n} \cos \theta+y_{n} \sin \theta\right)\right],
$$

where $k=2 \pi / \lambda$, and $G_{n}(\theta)$ and $\left(x_{n}, y_{n}\right)$ are the complex gain pattern and location of the $n$th element, respectively. The azimuth angle $\theta$ is measured from the positive $x$-axis in the anti-clockwise direction. Suppose the interpolated ULA has $M$ elements and is aligned along the $y$-axis. The $p$ th component of its array response vector $\mathbf{b}(\theta), p=1, \ldots, M$, is given by

$$
b_{p}(\theta)=\exp [j k d(p-1) \sin \theta],
$$

where $d$ is the inter-element spacing of the ULA

Suppose the planar array receives $L$ narrowband signals, $s_{1}(t), \ldots, s_{L}(t)$, each arriving from a distinct direction $\theta_{1}, \ldots, \theta_{L}$. The array output vector is given by

$$
\mathbf{x}(t)=\mathbf{A s}(t)+\mathbf{n}(t),
$$

where $\mathbf{A}=\left[\begin{array}{lll}\mathbf{a}\left(\theta_{1}\right) & \cdots & \mathbf{a}\left(\theta_{L}\right)\end{array}\right], \quad \mathbf{s}(t)=\left[\begin{array}{lll}s_{1}(t) & \cdots & s_{L}(t)\end{array}\right]^{T}, \quad \mathbf{n}(t)=$ $\left[\begin{array}{lll}n_{1}(t) & \cdots & n_{N}(t)\end{array}\right]^{T}, n_{n}(t)$ is the noise output of the $n$th sensor (assumed white, circular complex Gaussian and i.i.d. across the elements), and $\mathbf{n}(t)$ and $\mathbf{s}(t)$ are assumed to be stationary, zero mean, and uncorrelated with each other. The linear transformation on the output of the array is given by

$$
\mathbf{y}(t)=\mathbf{T} \mathbf{x}(t),
$$

where $\mathbf{T}$ is the $M \times N$ transformation matrix. Note that $\mathbf{T}$ will color the sensor noise at the interpolated array output. Hence, pre-whitening is required before we apply any DOA algorithms. Interested readers are referred to [6], [4], [7], [14] and [15], respectively, for the Bronez, Friedlander, Pesavento, Hyberg and Bühren formulations to find $\mathbf{T}$.

\section{PROPOSED APPROACH}

\subsection{Problem Formulation}

The principal idea of the new formulation is to approximate the array response vector of the interpolated array to that of a ULA within the in-sector and concurrently control its response over the entire out-of-sector region. The weighted least-squares (WLS) formulation is given as follows

$$
\min _{\mathbf{T}} \int_{-\pi}^{\pi} W(\theta)\|\mathbf{T a}(\theta)-s(\theta) \mathbf{b}(\theta)\|^{2} d \theta
$$

where $W(\theta)$ is the weighting function and $s(\theta)$ shapes the

\footnotetext{
${ }^{1}$ Although the proposed approach can be generalized to the elevation plane [4], for convenience, all signals are assumed to be in the azimuth plane of the array.
}

ULA response. The WLS solution to $(\mathcal{P} 5)$ is well known. The design of the shaping function $s(\theta)$ is arbitrary, provided the following requirements are met: (i) An ability to suppress out-ofsector signals to allow sector-by-sector processing to proceed satisfactorily; and (ii) $s(\theta)=1$ in the in-sector region, i.e., the in-sector response should approximate that of a ULA.

Clearly, the rectangular function will satisfy the above requirements but the discontinuity at the sector edges will result in large transformation errors. A better ploy is to roll-off smoothly the response from the edges of the in-sector to the edges of the image sector such as that defined by the raised cosine function

$$
s(\theta)= \begin{cases}1, & |\theta| \leq \theta_{0} \\ \frac{1}{2}\left\{1+\cos \frac{\pi\left(|\theta|-\theta_{0}\right)}{2 \theta_{0}-\pi}\right\}, & \theta_{0}<|\theta| \leq\left(\pi-\theta_{0}\right), \\ 0, & \left(\pi-\theta_{0}\right)<|\theta| \leq \pi\end{cases}
$$

where $\Delta \theta=\left[-\theta_{0}, \theta_{0}\right]$ is the in-sector region. The raised cosine shaping function was found to give a good overall error performance for different in-sector sizes.

It should be noted that the idea of controlling the out-ofsector response can also be formulated in other ways, e.g. as a minimax problem with constraints on the in-sector error. The weighted least squares formulation $(\mathcal{P} 5)$ is considered here because of its simplicity.

\subsection{Interpolated Array}

As a rule of thumb, the elements of the interpolated array should be placed in close proximity to the actual array [4]. For a UCA, the ULA can be placed along the diameter of the UCA with the two end elements on the circumference of the UCA.

Once the aperture of the ULA is fixed, we next determine the number of elements in the ULA. First, we note that $M$ is bounded from below by the requirement that $d / \lambda<0.5$. This condition arises from the fact that the proposed formulation has an extended roll-off region and the condition is required to prevent spatial aliasing. We next note that it might be desirable to have a large $M$ to increase the degrees of freedom. However $M$ cannot be too large since this will increase the condition number of $\mathbf{T}$ as the rows of $\mathbf{T}$ become more linearly dependent; and as discussed in [4], an ill-conditioned $\mathbf{T}$ should be avoided.

\section{NUMERICAL EXAMPLES}

In this section, we evaluate and compare the performances of the Friedlander, Pesavento, and our proposed formulations. The DOA estimator is MUSIC with forward-backward spatial smoothing (FBSS) [2] and sector-by-sector processing. The array is a UCA with radius $r=1.913 \lambda$ and $N=30$ elements. The interpolated ULA has $M=11$ and an in-sector size of $72^{\circ}$.

For the Pesavento formulation [7], the out-of-sector sidelobe constraint is set to $15 \mathrm{~dB}$ to ensure adequate suppression of the out-of-sector response. This corresponds to a $30 \mathrm{~dB}$ attenuation in the power response. We set the roll-off (or "don't care") region $^{2}$ on each side of the in-sector to $30^{\circ}$ to ensure a smooth

\footnotetext{
${ }^{2}$ Even though Pesavento et al. do not specifically mention a rolloff region in their formulation, it must exist since optimization is performed at discrete angles and the interpolation error can grow unacceptably large if the roll-off region is too narrow.
} 
roll-off and a better-conditioned T. The number of grid points chosen for the in-sector and out-of-sector regions are 30 and 100 , respectively, and the minimax criterion [7] is invoked.

Fig. 1 shows the beampattern of the interpolated ULA as well as the magnitude response of each interpolated array element for the Friedlander formulation. As can be seen, as a result of ignoring the out-of-sector response, the Friedlander formulation can suffer from severe distortion in this region. The phase response of each interpolated array element can also be shown to be severely distorted in this region. However, note that the rolloff is not immediate and there is a region beyond the $\pm 36^{\circ}$ edges of the in-sector region where the magnitude and phase responses remain close to the target ULA response.

Fig. 2 shows the beampattern and the magnitude response of each interpolated array element for the Pesavento formulation. It is clear that good suppression of the out-of-sector response is obtained beyond the roll-off region. However, in the roll-off region, the gain is still significant and thus phase distortions in this region can distort DOA estimation (see Fig. 3).

In the first study, we consider for the three formulations, the performance of MUSIC with FBSS. The UCA is oriented with an element at $0^{\circ}$. The signal scenario consists of three correlated signals, each with an SNR of $10 \mathrm{~dB}$ and arriving from $-5^{\circ}, 5^{\circ}$ and $30^{\circ}$ (all in the in-sector). The correlation coefficient between the first signal and the second or third signal is $e^{j \pi / 6}$, while it is 1 between the second and the third signal. For FBSS, three subarrays and the exact covariance matrices are used. We use $W(\theta)=1$ in $(\mathcal{P} 5)$. The multi-sector MUSIC spectra with the three formulations are given in Fig. 4. As expected, when all correlated signals are in the in-sector region, the three formulations can resolve the signals correctly.

In the second study (see Fig. 5), we repeat the first study, but with the third signal moved from $30^{\circ}$ to $50^{\circ}$, i.e., to the roll-off region. As can be seen, both the Friedlander and proposed formulations are able to resolve all DOAs accurately while the Pesavento formulation failed to resolve the closely spaced signals at $5^{\circ}$ and $-5^{\circ}$. The out-of-sector signal at $50^{\circ}$ has a negligible impact on the Friedlander formulation since at $50^{\circ}$, the response of this formulation is still well behaved (see Fig. 1). In contrast, the Pesavento formulation failed because, as can be seen from Figs. 2 and 3, the phase response at $50^{\circ}$ is severely distorted while the magnitude response is still significant.

In the third study (see Fig. 6), we repeat the second study but with the third signal moved from $50^{\circ}$ to $70^{\circ}$, which is just outside the roll-off region. Again, the proposed approach can resolve all signals correctly. However, we now see a reverse in trend between the Friedlander and Pesavento formulations. This is because the out-of-sector signal now falls in the badly distorted region for the Friedlander formulation, while it is effectively attenuated in the Pesavento formulation.

Though not shown, we report here that all three formulations were able to resolve the three signals correctly in the above three studies if they are mutually uncorrelated. This confirms the earlier finding that out-of-sector uncorrelated signals have only a minor impact on the performance of in-sector DOA estimation [4],[10]. In addition, we note that all three formulations gave biased DOA estimates because of the transformation error. In particular, depending on the choice of array and design parameters, the proposed formulation may give larger biases than the other two formulations. This can be remedied by modifying $(\mathcal{P} 5)$ to incorporate constraints to limit the transformation error.

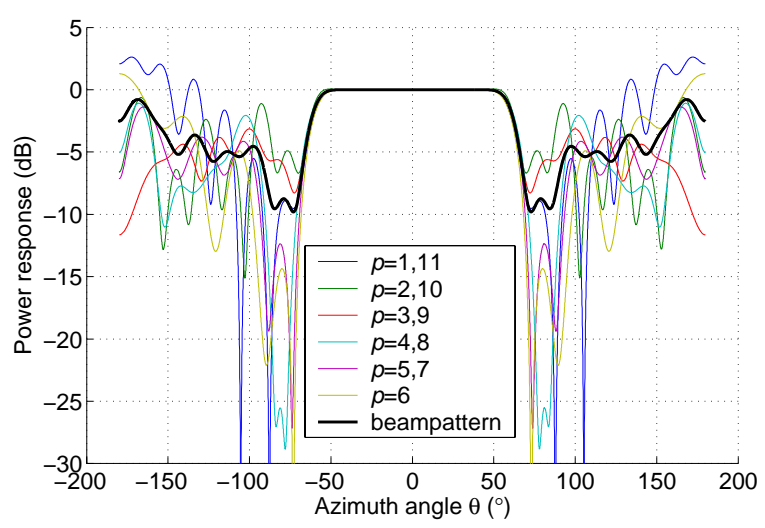

Fig. 1. Power response of the Friedlander formulation for each of the 11 virtual elements. Five pairs of curves overlap due to symmetry.

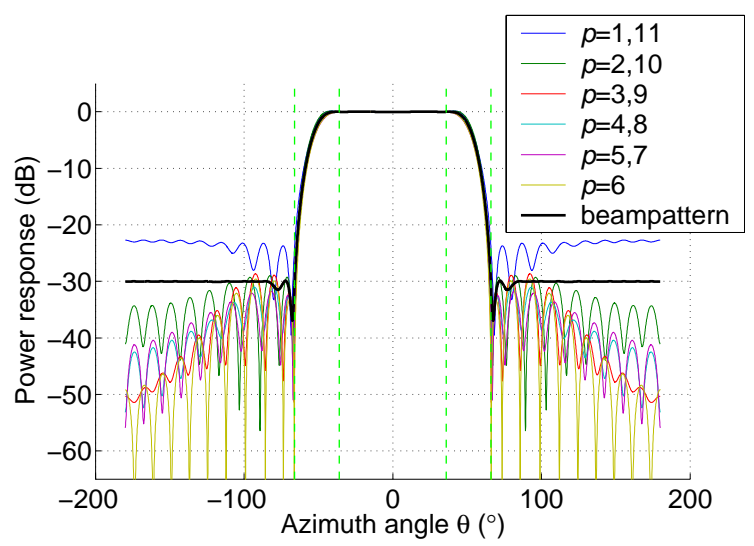

Fig. 2. Power response of the Pesavento formulation for each of the 11 virtual elements. Five pairs of curves overlap due to symmetry. Edges of roll-off regions marked by vertical dashed lines.

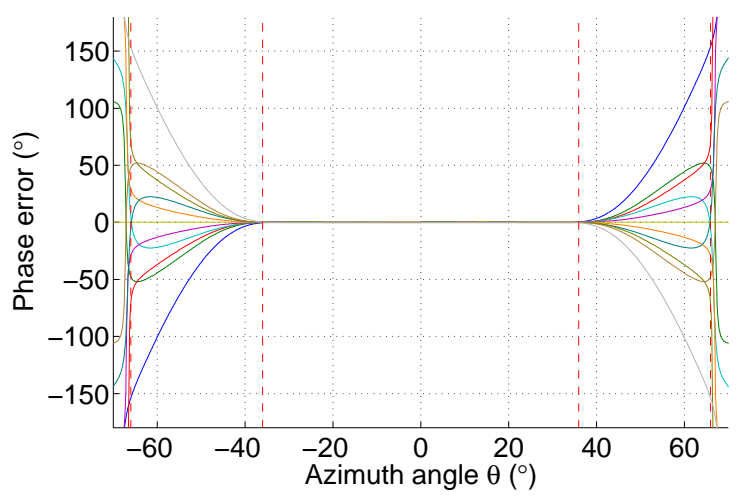

Fig. 3. Phase error of the Pesavento formulation for each of the 11 virtual elements. Edges of roll-off regions marked by vertical dashed lines.

Finally, we emphasize that while the above numerical studies give the comparative performances of the different formulations 
for the array interpolation approach, the main thrust is to demonstrate the limitations in the existing formulations and highlight the ability of our proposed formulation to deal with a general signal scenario.

\section{CONCLUSIONS}

Array interpolation can greatly simplify array signal processing for arbitrary planar arrays. However, the existing formulations can have problems with coherent out-of-sector signals interfering with the DOA estimation of the in-sector signals. We proposed a new formulation to take into account the array response over the entire azimuth and confirmed with numerical examples the effectiveness of our approach in mitigating the malevolent influence of coherent out-of-sector signals.

\section{REFERENCES}

[1] A. J. Barabell, "Improving the resolution performance of eigenstructure-based direction-finding algorithms," in Proc. IEEE ICASSP'83, vol. 1, pp. 336-339, Boston, MA, May 1983.

[2] S. U. Pillai and B. H. Kwon, "Forward/backward spatial smoothing techniques for coherent signal identification," IEEE Trans. Acoust., Speech and Signal Processing, vol. 37, pp. 8-15, Jan. 1989.

[3] P. Stoica and K. Sharman, "Novel eigenanalysis method for direction estimation," IEE Proc., vol. 137, pt. F, pp. 19-26, Feb. 1990.

[4] B. Friedlander, "The Root-MUSIC algorithm for direction finding with interpolated arrays," Signal Processing, vol. 30, pp. 15-29, Jan. 1993.

[5] K. M. Reddy and V. U. Reddy, "Analysis of interpolated arrays with spatial smoothing," Signal Processing, vol. 54, pp. 261-272, Nov. 1996.

[6] T. P. Bronez, "Sector interpolation of nonuniform arrays for efficient high resolution bearing estimation," in Proc. IEEE ICASSP'88, vol. 5, pp. 2885-2888, New York, NY, Apr. 1988.

[7] M. Pesavento, A. B. Gershman, Z. Q. Luo, "Robust array interpolation using second-order cone programming," IEEE Signal Processing Lett., vol. 9, pp. 8-11, Jan. 2002.

[8] B. Friedlander and A. J. Weiss, "Direction finding using spatial smoothing with interpolated arrays," IEEE Trans. Aerosp. Electron. Syst., vol. 28, pp. 574-587, Apr. 1992.

[9] A. J. Weiss and M. Gavish, "Direction finding using ESPRIT with interpolated arrays," IEEE Trans. Signal Processing, vol. 39, pp. 1473-1478, Jun. 1991.

[10] A. J. Weiss, B. Friedlander, and P. Stoica, "Direction finding with interpolated MODE," in Proc. IEEE 27th Asilomar Conf. Signals, Syst., Comput., vol. 2, pp. 13761380, Pacific Grove, CA, Nov. 1993.

[11] A. B. Gershman, "Direction finding using beamspace root estimator banks," IEEE Trans. Signal Processing, vol. 46, pp. 3131-3135, Nov. 1998.

[12] D. V. Sidorovich and A. B. Gershman, "Two-dimensional wideband interpolated Root-MUSIC applied to measured seismic data," IEEE Trans. Signal Processing, vol. 46, pp. 2263-2267, Aug. 1998.

[13] J. F. Sturm, "Using SeDuMi 1.02, a MATLAB toolbox for optimization over symmetric cones," Optim. Meth. Softw., vol. 11-12, pp. 625-653, Aug. 1999.

[14] P. Hyberg, M. Jansson, B. Ottersten, "Array mapping: optimal transformation matrix design," in Proc. IEEE ICASSP'02, vol. 3, pp. 2905-2908, Orlando, FL, May 2002.

[15] M. Bühren, M. Pesavento, and J. F. Böhme, "A new approach to array interpolation by generation of artificial shift invariances: interpolated ESPRIT," in Proc. IEEE ICASSP'03, vol. 5, pp. 205-208, Hong Kong, China, Apr. 2003.

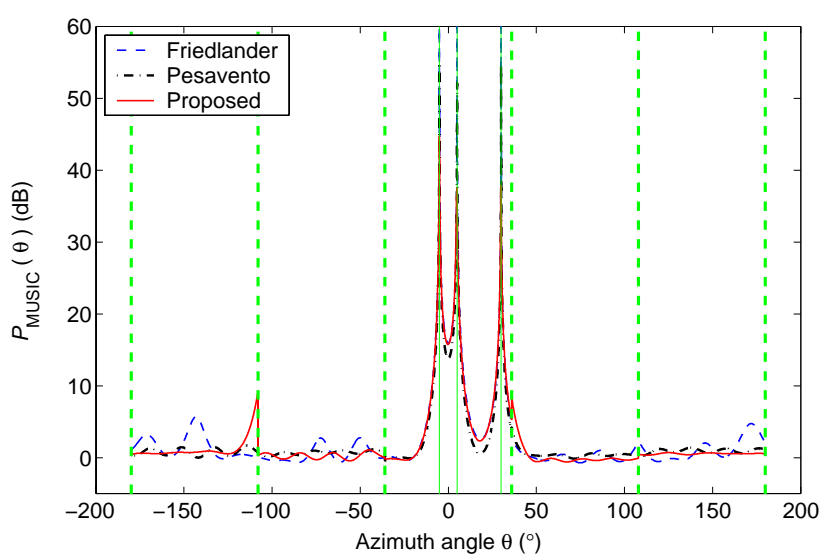

Fig. 4. Multi-sector MUSIC spectra for the first study

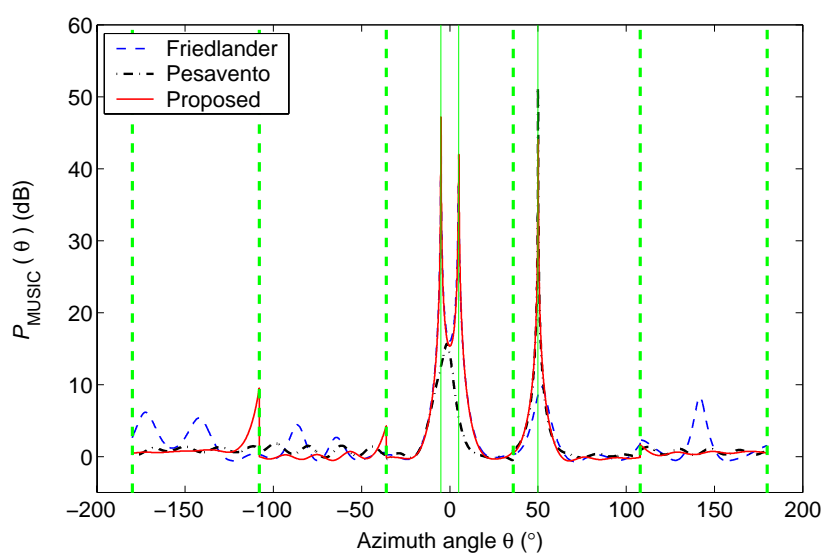

Fig. 5. Multi-sector MUSIC spectra for the second study

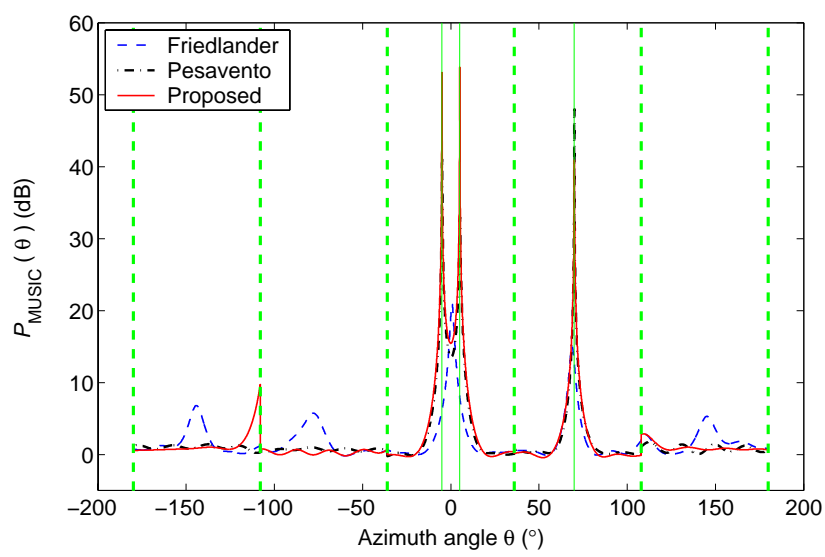

Fig. 6. Multi-sector MUSIC spectra for the third study. 